\title{
Need-based transfers on a network: A model of risk-pooling in ecologically volatile environments
}

Running headline: Need-based transfers on a network

YAN HAO ${ }^{1,2}$, DIETER ARMBRUSTER ${ }^{1}$, LEE CRONK, C. ATHENA AKTIPIS $4,5^{*}$

1- School of Mathematical and Statistical Sciences, Arizona State University

2- Department of Mathematics and Computer Science, Hobart and William Smith Colleges

3- Department of Anthropology, Rutgers University

4- Department of Psychology, Arizona State University

5- Center for Evolution and Cancer, University of California, San Francisco

*Corresponding Author

Department of Psychology

Arizona State University

P.O. Box 871104

Tempe, AZ 85287-1104

aktipis@asu.edu

415.843.1793

Word Count: 6,567 


\begin{abstract}
Need-based transfers are a widespread form of human cooperation across cultures that enhance survival in marginal environments. Examples include central place food sharing among foragers and stock friendships among pastoralists. Previous models have demonstrated that such systems lead to higher rates of herd survival under volatile ecological conditions, such as those experienced by the Maasai of East Africa. The Maasai use a need-based transfer system called osotua that leads to risk pooling. Here we implement osotua-style asking and giving rules on a network in order to understand which network features promote herd survival. We find that (1) greater network size increases herd survival when individuals selectively ask their wealthiest partner for livestock but not when they ask a partner at random, (2) greater network connectedness improves herd survival regardless of whether individuals ask their wealthiest partner or ask a partner at random, (3) greater network heterogeneity leads to higher herd survival with selective asking of wealthy partners and decreases herd survival for random asking. In general, selective asking of wealthy partners is associated with higher rates of herd survival. We also examined the features of survival networks in order to understand the characteristics of the networks that result from 50 simulated years of osotua-style sharing under ecologically volatile conditions and the elimination of individuals who do not stay above sustainability threshold. These results will help inform further fieldwork on the need-based transfer systems and increase our understanding of features of sharing networks that enable risk pooling. Simple decentralized sharing rules can be highly effective for pooling risk, suggesting that complex cultural institutions may not be necessary for expansion into ecologically marginal environments.
\end{abstract}

Keywords: risk pooling, sharing, cooperation, social insurance.

\title{
1 Introduction
}

Natural disasters, disease, theft, and a wide variety of other events constitute common but inherently unpredictable threats to human wellbeing and survival. In a word, they create risk. Risk has been defined in a variety of ways, but in human behavioral ecology it refers to "unpredictable variation in an outcome with consequences that matter" (Winterhalder 
2007:433). The problem of how to effectively manage risk is therefore a recurrent pressure shaping the evolution of human behavior and cultural institutions, including those surrounding resource transfer and cooperation. Humans are remarkable for their ability to adapt not only to the environment in which they originally evolved but also to a wide variety of marginal, ecologically volatile environments around the world. One of the keys to our ancestors' success in this regard may have been their ability to effectively deal with the risks and uncertainties associated with such environments through systems of resource transfer.

People throughout history and around the world have developed a variety of strategies for dealing with risk. Four common risk management strategies are risk retention, risk avoidance, risk reduction, and risk transfer (Dorfman, 2007). Risk retention consists of accepting risk and absorbing any resulting losses. When people store resources in anticipation of future shortages and when institutions self-insure, they are engaging in risk retention. Risk avoidance involves the reduction of one's dependence on high variability outcomes. For example, working for a steady, reliable paycheck rather than as a freelancer is a way of avoiding risk. Risk reduction includes efforts to lower the probability of loss, or, alternatively, to reduce the size of losses. Investors reduce risk by buying bonds as well as stocks. Finally, risk transfer is the exchange of risk from one individual or group to another. Buying an insurance policy is one common way to transfer risk. This article concerns another common type of risk transfer: risk pooling, also known as risk sharing (e.g., Barr \& Genicot, 2008; Fafchamps \& Lund, 2003). Risk pooling refers to agreements to take on some of another party's risk in exchange for their willingness to take on some of one's own (Wiessner, 1982; Cashdan, 1985).

Risk transfer (including risk pooling) is the only one of these four risk management strategies that necessarily involves cooperation. Typically, risk pooling arrangements entail a commitment to helping a risk-pooling partner when that partner is in need and one is able to help. Because the donor's response to the recipient's need is the defining feature of such systems, we refer to them as systems of risk-pooling through need-based transfers.

In the context of foragers, need-based transfers bear some resemblance to the concepts of tolerated theft (Blurton Jones, 1984, 1987; see also Isaac, 1978; Jaeggi and Van Schaik, 2011) and demand sharing (Peterson 1993) in that all three involve transfers from those who are at least temporarily resource-rich to those who are in need. In tolerated theft and demand 
sharing, one shares in order to avoid the costs associated with defending resources. Those costs may sometimes include gaining a reputation for being stingy. In contrast, within needbased transfers like the osotua system which we modeled here, there are potential benefits to creating and maintain relationships with partners on whom one can depend if one is in need in the future. Need-based transfer systems such as osotua can help to mitigate the risks associated with living in marginal or unpredictable ecological conditions (Aktipis et al., 2011). In some ways, need-based transfers can be considered a larger category of resource transfers that include tolerated theft and demand sharing as well as resource transfers that occur as part of the osotua system and related systems. These types of resource transfers fall on a continuum from highly coercive (e.g., theft or extortion) to voluntary transfers (e.g., osotua transfers or charitable gifts). We use the term 'need-based transfers' in this paper to mean voluntary need-based transfers that can serve a risk pooling function.

Risk pooling through need-based transfer arrangements increase the likelihood that both parties will suffer losses while decreasing the severity of those losses. In situations where losses tend to be both severe and unpredictable, accepting a predictable but relatively small cost while avoiding large, unpredictable ones can be a beneficial tradeoff for all concerned. Among hunter-gatherers, for example, big game is a resource that is usually acquired unpredictably. This means that many would-be hunters actually come home empty-handed much of the time. If they had to rely solely upon their own foraging efforts, they would either starve or abandon big game hunting in favor of more reliable, predictable food sources, such as plants, honey, and small game. A solution to this problem practiced by hunter-gathering peoples around the world is to pool risk by having the members of a group forage independently followed by an equitable division of what they have acquired (Winterhalder, 1986, 1990; Wiessner, 1982; Cashdan, 1985; Gurven \& Hill, 2009; Gurven et al., 2000, 2004; Price, 1962; Price, 1975; Woodburn, 1998). Although food sharing results in risk pooling through the pooling of resources, risk can be pooled even when those who own resources retain control of them. Consider, for example, the hxaro gift-giving system of the Ju/hoansi of Namibia and Botswana (Wiessner, 1977; Wiessner, 1982; Wiessner, 2002). Hxaro gifts, which are typically small items such as beads and arrowheads, are essentially tokens used to establish and maintain relationships that allow hxaro partners to call upon each other when in dire need of food or water. The hxaro system 
thus creates a risk-pooling network beyond just the local groups within which large game is routinely shared.

Need-based transfers that lead to risk-pooling also occur in food-producing societies. In one simulation of such a system among early twentieth century Hopi, Hegmon (1989) showed that such sharing enhances survival most when households share with those in need only after having first met their own needs rather than by pooling all food harvested. Similarly, many pastoralists also commonly pool risk not by pooling resources but rather by transferring limited numbers of livestock to those in need (Almagor, 1978; Bollig, 1998; Bollig, 2006; DeVries et al., 2006; Dyson-Hudson, 1966; Gerkey, 2010; Gulliver, 1955; Flannery et al., 1989; McCabe, 1990; Potkanski, 1999). With a few exceptions (e.g., Bollig, 1998, 2006), such systems have been documented only qualitatively, with a focus mainly on the rules and expectations that govern them, with scant attention being paid to such quantifiable matters as numbers of stock-friendships maintained or numbers of head transferred. Along with our previous simulation described below (Aktipis et al., 2011), this article is a first step in a broader effort to understand such systems by using computer simulations to generate predictions that we and our collaborators will in the future test at field sites.

As a model of adaptive decision-making by pastoralists in the face of risk, our work follows in a long tradition of such models. These include Mace's models of Gabbra decision-making regarding herd management and birth scheduling (Mace and Houston 1989, Mace 1993, Mace 1996), Flannery et al.'s computer simulation of herd dynamics among Andean pastoralists (Flannery et al., 1989), and De Vries et al.'s (2006) simulation of the impact of livestock acquisition patterns on herd demography. Our contribution to this tradition is in the use of computer simulations to examine pastoralists' decisions about whether to ask for or give livestock to one another.

The inspiration for our model comes primarily from our understanding of a system of riskpooling through need-based transfers among Maa-speaking pastoralists in East Africa such as the Maasai and Samburu, which they refer to by their word for umbilical cord: osotua. Osotua relationships are started in many ways, but they usually begin with a request for a gift or a favor. Such requests arise from genuine need and are limited to the amount actually needed. Osotua does not follow a schedule, and it will not go away even if much time passes 
between gifts. Although osotua involves a reciprocal obligation to help if asked to do so, actual osotua gifts are not necessarily reciprocal or even roughly equal over long periods of time. The flow of goods and services in a particular relationship might be mostly or entirely one-way, if that is where the need is greatest. Maasai interviewees state very clearly that osotua gifts neither create nor repay debt, that osotua gifts are not payments at all, and that it is therefore inappropriate to use the verb to pay (alak) when referring to them. Osotua imbues respect (enkanyit), restraint, and a sense of responsibility in a way that non-osotua economic relationships do not. In the words of one interviewee, keiroshi: It is heavy.

To learn more about osotua, Cronk (Cronk, 2007; Cronk \& Wasielewski, 2008) used the osotua norm to frame Trust Games played by Maasai. In the Trust Game, two players, who are anonymous to each other, are given an initial endowment. The first player can then give none, some, or all of his endowment to the second player. The experimenter triples that amount and then passes it on to the second player. Thus, the second player has an endowment that consists not only of the amount provided initially by the experimenter but also the amount given by the first player, multiplied by three. The second player can then give some, none, or all of the funds in his control to the first player. A total of 50 games were played. All players were given standard instructions, in Maa, on how to play the Trust Game. Half of the games were played with no framing beyond the instructions themselves and the other half were told "This is an osotua game." The osotua framing led to lower amounts given and lower amount expected (compared to the unframed game). In addition, osotua framing led to a negative correlation between the amounts given and received, in contrast to a positive correlation in the unframed games. One possible interpretation of this is that second players in the framed games may have seen low amounts given as a signal of need on the part of the first player. These findings suggest that the osotua framing shifts game play away from the logic of investment, debt, and repayment and towards the logic of risk-pooling through need-based transfers: Osotua partners have a mutual obligation to respond to one another's genuine needs, but only with what is genuinely needed.

Osotua and other risk-pooling systems among pastoralists may be facilitated by certain characteristics of livestock themselves. First, livestock are a volatile form of wealth. Although herds have the capacity to grow, they can also be rapidly, severely, and unpredictably reduced 
by diseases, droughts, and theft (Bollig, 1998; Dahl \& Hjort, 1976). Any pastoralist would do well to find ways to reduce his or her exposure to risk. One way to understand the relationship between livestock and osotua relationships is to see them as different kinds of "wealth": livestock are "material wealth" and osotua relationships are "relational wealth" (Borgerhoff Mulder and Beheim 2011). Both types of wealth may range from very stable to very volatile. While livestock are a volatile form of material wealth, osotua relationships are designed to be an extremely stable type of relational wealth. Binding the latter to the former helps mitigate the former's volatility.

Second, livestock are a form of wealth that is visible to the entire community. Unlike money, livestock cannot be secreted away. Although the lending of livestock among herders means that a glance at a herd-owners' pens at the end of the day will not yield a perfect estimate of his wealth, such lending involves too small a portion of herds to throw such estimates off by very much. If a man has lots of livestock in his pens at sundown, then he is very unlikely to be poor; if he has few livestock in his pens at sundown, then he is very unlikely to be rich. It is therefore easy to see whether an individual is in good or poor economic condition and thus whether they are being truthful about their need for additional livestock and their ability provide livestock to others. Although the avoidance of cheating and cheaters is an important theme in the study of cooperation (e.g., Cosmides \& Tooby 2005), the public nature of livestock wealth makes osotua one system in which cheating by feigning poverty is unlikely to be a major concern.

To explore the role that osotua relationships play in the management of risk among Maaspeaking pastoralists, Aktipis et al. (2011) developed an agent-based model involving simulated pairs of osotua partners. Herd survival was defined in terms of the agents' success at keeping their herds above a critical threshold that we derived from the literature on herd demography and household food needs among East African pastoralists (Dahl and Hjort, 1976). Agents with herds that dropped below the threshold for two consecutive rounds were removed from the simulations, along with their herds (see Figure 3). This is consistent with the pattern seen among the Maasai in which impoverished families become relegated to a denigrated and shunned social category called il-torrobo (Galaty 1982, Cronk 2004). Although il-torrobo typically engage in such non-pastoralist pursuits as hunting, gathering, and bee-keeping in 
order to stay alive, they do not abandon whatever few animals they might still own.

Exchange between partners based on the rules of osotua relationships led to better herd survival than simulations with no exchange or with exchange based on probabilistic rules. Correlations between partners' herd survival rates were also stronger in simulations with exchanges based on the osotua rules, indicating that osotua exchange partners were indeed achieving their higher rates of survival through increased risk pooling. Aktipis et al. (in prep.) have extended this agent-based model by comparing the performance of players following the rules of osotua exchange (i.e., risk-pooling) to partners following the logic of account-keeping reciprocity. As with the previous simulation, partners whose behavior is guided by the rules of need-based transfers do better than those whose behavior is guided by the rules of reciprocity in the strict sense of balance, account-keeping, and tit-for-tat. In this paper, we extend this research in a new direction by looking at need-based transfers in the context of social networks. We also compare two types of need-based transfer rules, one of random asking among individuals in a network of partners and another of selective asking of the wealthiest partner. It is currently not known which specific rules individuals use for deciding who to ask for help within the osotua system and similar need-based transfer systems. We explore these variations of the rules within different social networks in order to guide subsequent fieldwork and experiments with human subjects.

\section{Model and Methods}

\section{$2.1 \quad$ Network generation}

To create a baseline for comparison, we begin by studying the average herd survival in homogeneous osotua gift-giving networks. By homogeneous we mean that all individuals in the network have the same number of osotua partners. As the diagram in Fig. 2 shows, each vertex represents an individual and each pair of osotua partners is connected by an edge. We will use $N$ to represent the network sizes i.e. the number of individuals (vertices) in a network. We will use $k$ to represent the average degree, i.e. the average number of osotua partners per

individual. Because the osotua partnerships are bidirectional, to guarantee the symmetry of 
the network, the values of $k$ are limited to even numbers. For example, in Fig. 2 (A) each node $i,(1 \leq i \leq N)$ has degree four $\left(d_{i}=4\right)$ and is connected to two of its closest neighbors from both sides.

The network dynamics of a heterogeneous network was studied using Watts-Strogatz random networks (Watts \& Strogatz 1998). The Watts and Strogatz networks provide a simple algorithm for generating networks that have systematically varying levels of heterogeneity. Each random network is generated by randomly rewiring a fraction $(\beta)$ of the edges of a homogeneous network. To keep the average degree of the network $k$ unchanged, adding or deleting edges is not allowed in this process. As shown in Fig. $2(\mathrm{~B})$ and $(\mathrm{C})$, as $\beta$ increases, the number of edges to be rewritten increases proportionally which increases the randomness of the network. Consequently, $\beta$ can be used to quantitatively measure the randomness of a heterogeneous network.

\subsection{Implementation of osotua gift-giving on a network}

To be consistent with the previous study on osotua gift-giving in pairs (Aktipis et al. 2011), we formalized the osotua rules on a network as follows:

1. Osotua asking rule: Individuals make a request for cattle only if their current holdings are below the asking threshold (the minimum herd size of 64). Each individual is allowed to make only one request per year.

2. Osotua giving rule: Individuals give what is asked, but not so much as to put cattle holdings below their own giving threshold (also the minimum herd size of 64 ). If asked, an individual may give multiple gifts.

3. Asking order: If multiple individuals make requests, requests are processed one at a time based on a randomly selected order.

\section{Asking strategy:}

(a) Random asking: Assuming that individuals do not have information on their partners' herd sizes, individuals make a request to one of their osotua partners with equal probability. 
(b) Selective asking: Assuming that individuals have information on their partners' herd sizes, individuals pick the wealthiest among all their osotua partners to make a request. When two or more of an individual's partners have the same maximum herd size, he will make a request to only one of them with equal probability.

\subsection{Simulation}

Herd survival in osotua networks was simulated using Matlab. As shown in Fig. 3, each simulation consists of five phases: Initialization, Random growth, Disasters, Osotua request, Osotua response, and Vitality check. The default network size in all simulations is $N=100$ vertices and the average degree is $k=4$, unless otherwise specified. In the simulations, the

herd size $H_{i}^{(n)}$ of each individual $i(1 \leq i \leq N)$ at year $n$, is updated at the end of year $n$ simultaneously and are tracked until $n=50$. For every network topology, simulations are repeated 10000 times and the average values are reported.

\subsubsection{Initialization}

Each network topology is specified by an $N \times N$ adjacency matrix $A=\left\{a_{i j}\right\}$. Because the osotua obligation is reciprocal, the osotua network is undirected, i.e., if node $i$ is an osotua partner to node $j$ then $a_{i j}=a_{j i}=1$. Consequently, the adjacency matrix $A$ is a symmetric matrix with row sum $k$. Note that for a homogeneous network, $A$ has permutation symmetry (each node is identical) and additional symmetries depending on the structure of the edges of the network. To generate a Watts-Strogatz network with a given $\beta$, we randomly pick $\frac{\beta N k}{2}$ edges, disconnect one end and reconnect the link to a different randomly picked node. To implement this, $\frac{\beta N k}{2}$ pairs of subscripts $(i, j)$ are randomly selected from $\{(i, j) \mid A(i, j)=1\}$ with equal probability. Then, a $\hat{j} \neq j$ is randomly selected to replace $j$ for each pair. The new adjacency matrix $\hat{A}$ will lose the permutation symmetry but the row sums are kept unchanged.

For both homogeneous and random network, initial herd sizes are set to

$$
H_{i}^{(0)}=70, \quad 1 \leq i \leq N
$$

for all individuals at the beginning of each simulation. 


\subsubsection{Random growth}

Each year, an individual herd $i$ grows at a random rate $g_{i}^{(n)}$ that is sampled from a Gaussian distribution. Consistent with Aktipis et al. (2011), the growth rate distribution is given by

$$
g_{i}^{(n)} \sim \mathcal{N}\left(3.4 \%,(2.53 \%)^{2}\right) \quad 1 \leq i \leq N, 1 \leq n \leq 50
$$

i.e. the growth rate averages at $3.4 \%$ with a standard deviation of $2.53 \%$, a typical annual growth rate for cattle herds in this region of East Africa (Dahl \& Hjort, 1976). Consequently, before taking disasters into account, the herd size for individual $i$ at the end of year $n$ should be

$$
\tilde{H}_{i}^{(n)}=H_{i}^{(n-1)}+H_{i}^{(n-1)} g_{i}^{(n)}, \quad 1 \leq i \leq N
$$

With a very small probability the Gaussian distribution will give us negative numbers, which we recognize them as negative growth. This is consistent with the fact that herds can decrease not only due to catastrophes such as drought, disease, and theft, but also simply because in some years deaths may slightly exceed births.

\subsubsection{Disasters}

The sequence of disasters is assumed to be generated by Poisson processes: the time until the next disaster strikes is exponentially distributed with mean $10 \%$. Each individual herd is considered independent and is assigned a disaster calendar at the beginning of a simulation which is a series of exponentially distributed random numbers indicating gaps between disasters. If year $n$ is a disaster year for individual $i$, a random number $l_{i}^{(n)} \sim \mathcal{N}\left(30 \%,(10 \%)^{2}\right)$ is drawn to decide the percentage of the herd that is lost in this year. At the end of each year, the disaster phase checks every individual's calendar: if it is a disaster year for an individual, then the corresponding loss $\Delta(i, n)=l_{i}^{(n)} \tilde{H}_{I}^{(n)}$ is subtracted from that individual's herd size. In general, after growth and loss

$$
\tilde{\tilde{H}}_{i}^{(n)}=\left\{\begin{array}{lr}
\tilde{H}_{i}^{(n)}-\Delta(i, n) & \text { disaster year } \\
\tilde{H}_{i}^{(n)} & \text { no disaster year }
\end{array}\right.
$$




\subsubsection{Osotua request}

After accounting for random growth and disaster losses, the program will enumerate the individuals whose herd sizes fall below the sustainability threshold $(\theta=64)$ and randomly generate an order for them to make one request each. Every individual uses the same pre-determined strategy (random asking or selective asking) to make a request to one of his osotua partners, asking for enough to bring his herd size back to the sustainability threshold

$$
\alpha_{i}^{(n)}= \begin{cases}0 & \left(\tilde{\tilde{H}}_{i}^{(n)} \geq \theta\right) \\ \theta-\tilde{\tilde{H}}_{i}^{(n)} & \left(\tilde{\tilde{H}}_{i}^{(n)}<\theta\right),\end{cases}
$$

where $\alpha_{i}^{(n)}$ is the number of cattle asked by individual $i$. In each simulation, all individuals use the same asking strategy throughout the entire 50 years. Each individual is allowed to make only one request per year, even if no livestock are received as a result of the request.

\subsubsection{Osotua response}

Individuals respond to osotua requests one at a time following the order in which they are made. When an individual, $j$, is asked by individual $i, j$ will respond by giving $\gamma_{j \rightarrow i}^{(n)}$ cattle to $i$ where

$$
\gamma_{j \rightarrow i}^{(n)}= \begin{cases}0 & \left(\tilde{\tilde{H}}_{j}^{(n)}<\theta\right) \\ \tilde{\tilde{H}}_{j}^{(n)}-\theta & \left(\tilde{\tilde{H}}_{j}^{(n)}<\theta+\alpha_{i}^{(n)}\right) \\ \alpha_{i}^{(n)} & \left(\tilde{\tilde{H}}_{j}^{(n)}>\theta+\alpha_{i}^{(n)}\right) .\end{cases}
$$

In other words, if $j$ has more cattle than $\theta$ he will respond to the request made by $i$. If $j$ has more than $\theta+\alpha_{i}^{(n)}$, he will fulfill the request made by giving $\alpha_{i}^{(n)}$ cattle to $i$, otherwise $j$ only give all his "extra" (the number that beyond threshold $\theta$ ) cattle to $i$.

\subsubsection{Viability check}

When the osotua phase finishes, the herd sizes are finalized for the current year,

$$
H_{i}^{(n)}=\tilde{\tilde{H}}_{i}^{(n)}+\sum_{j} \gamma_{j \rightarrow i}^{(n)}-\sum_{j} \gamma_{i \rightarrow j}^{(n)} .
$$


In the viability check phase the herd size of the current and the previous year are checked.

If an individual herd fails to meet the threshold for two consecutive years, i.e. $H_{i}^{(n)}<\theta$ and $H_{i}^{(n-1)}<\theta$, the herd (and the individual) will be removed from the simulation by setting $H_{i}^{(m)}=0$ for all $m>n$. This modeling assumption is based upon the fact that, among the Maasai, individuals whose herds are below sustainability threshold typically keep their remaining cattle but find other ways to make a living (e.g., hunting, gathering, bee-keeping) outside of the network of herders. The remaining herds will then restart a yearly cycle with the random growth phase (2.3.2) until the program ends after the fiftieth year.

\section{Results}

Previous work has shown that osotua gift-giving between two individuals enhances the survival of their herds (Aktipis et al., 2011). We extended this gift-giving strategy to a network in order to explore the effect of network characteristics on herd survival. Here we report the quantitative impact of varying the network size $(N)$, number of connections $(k)$, and network heterogeneity $(\beta)$. Further, we investigate how the network centrality and individual popularity measures associate with final herd survival (both for the initial networks and survival networks).

\subsection{Network size}

We study homogeneous $(\beta=0)$ networks exemplified by Fig. 2(A) and focus on the impact of the size of the network and the connectivity of the network on the average survival rate by the end of the fiftieth year to obtain benchmarks for non-homogeneous network topologies when $\beta \neq 0$, such as shown in Fig. 2(B,C).

To study whether larger network size promotes herd survival, we varied $N$ in homogeneous $(\beta=0)$ networks exemplified by Fig. 2A. $N$ was varied from 5 to 100 while the number of osotua partners per person was maintained at $k=4$. Fig. 4(A) shows that increasing the network size $(N)$ has no significant effect on herd survivorship when all the participants use a random asking strategy. When a selective asking strategy is used, however, increasing $N$ does enhance herd survival as shown in Fig. 4(B). This effect is most significant when the network size is relatively small $(N \leq 10)$. When the size of the network reaches 10 , further increases 
do not improve herd survivorship.

Comparing Fig. 4(A) to Fig. 4(B) we note that, for all network sizes, survivorship is always significantly $(\geq 20 \%)$ better for a selective asking strategy than for a random asking strategy. This difference can also be observed in the beginning and ending network topology figures shown in Fig. 5.

\subsection{Network connectedness}

Next, we examine the impact of increasing the number of connections on herd survival, while keeping the size of the network constant at $N=100$ and using a homogenous network. We double $\mathrm{k}$ in each successive simulation from $k=2$ up to $k=64$. Again, selective asking is significantly better than random asking at increasing herd survivorship. In addition, Fig. 6 shows that increasing the average node degree promotes herd survival for both asking strategies. Furthermore, when a selective asking strategy is used (Fig. 6B), this effect is more significant than when a random asking strategy is used (Fig. 6A). However, the increase of the survival rate with the increase of the degree saturates: increasing the number of connections ( $k$ ) above 32 does not improve herd survival, regardless of which asking rule is used.

\subsection{Network heterogeneity}

\subsubsection{Randomness and herd survival}

Finally, we examine the effect of network heterogeneity $(\beta)$ on herd survival while keeping the network size $(N=100)$ and connectedness $(k=4)$ constant. Here, network heterogeneity is the proportion of connections that are randomly rewired, and this is varied from 0.1 to 1 . One thousand Watts-Strogatz networks are randomly generated for each $\beta$, and simulations of each network through year 50 is repeated 1000 times. The average survival rates are averaged and displayed in Fig. 7, where the effect of heterogeneity parameter $\beta$ is shown clearly. Network heterogeneity affects herd survival differently depending on the asking strategy: When $\beta$ is relatively small and a random asking strategy is used (shown by blue dots) increasing network heterogeneity harms herd viability. On the other hand, when a selective asking strategy is used (black dots), heterogeneity improves the average network survival rate. These effects 
diminish when $\beta \geq 0.5$ for random asking and when $\beta \geq 0.7$ for selective asking. The reasons why network heterogeneity influences the network herd viability in two distinct directions will become clear after we see the individual survival dynamics, which is discussed in Sec 3.4.

\subsection{Degree distribution}

Fig. 8 shows that, within a heterogeneous network where the degree (number of connections) of individuals vary, the survival of an individual's herd increases with the number of connections. Similar to Fig. 6, this increase saturates at approximately four osotua partners for random asking and approximately twelve for selective asking. Again, when using the selective asking strategy, the increase is more significant. Hence these results indicate that most individuals will have a better chance to survive through 50 years if they increase their number of osotua partners. However, the diminishing improvements in survival as partners are added, as shown in Figure 8, suggests that there are limits to the degree to which additional partners are worth adding presumably because at some point the increase in herd survivorship one experiences by adding another partner is less than the social costs of doing so.

The different saturation levels for random and selective asking explain why network randomness $(\beta)$ influences these different strategies in distinct ways as shown in Fig.7. Heterogeneity of the networks leads to distribution of the initial degrees. As we can see from Fig. 8, the survival rate for random asking saturates at $k=4$ and hence individuals with degree higher than four all have the same survival rate. Hence the average survival rate over the degree distribution of a heterogeneous network for random asking will be lower than for a homogenous network with the same mean degree of $k \geq 4$.

For selective asking the survival rate continues to increase with the degree until $k \approx 12$. Hence for networks with a mean degree less than twelve, the average survival rate will be higher for heterogeneous networks than for homogeneous ones.

\section{Discussion}

Risk management is important in all human societies. However, because pastoralists typically live in volatile environments and are often beyond the easy reach of law enforcement, they 
may be in greater need of decentralized systems for managing risk and responding to shocks compared to people with other methods of subsistence. Although risk-pooling through needbased transfers is just one way to manage risk, it may be particularly well suited to situations in which resources are scarce and other risk management options are limited, as is the case with pastoralists.

This simulation is part of The Human Generosity Project (HGP), which aimsto understand the nature of human generosity,. The HGP investigates the role of need-based transfers in human cooperation through field work at multiple field sites among pastoralist groups in East Africa including Maasai in Kenya and Tanzania and Karimojong in Uganda. This project also includes simulation work focused on modeling need-based transfer systems with various ecological, social and demographic conditions. The findings from this model will guide fieldwork at each of these sites, and findings from the fieldwork will subsequently guide future simulations. The HGP will also conduct experiments on human subjects to explore the psychological bases for giving and use these findings to also guide fieldwork and future modeling.

Previous computer simulations have shown that the osotua risk pooling system used by Maasai pastoralists improves herd survivorship for dyads of livestock owners (Aktipis et al., 2011). In this article, we modeled that system on a series of simulated networks, varying the overall number of individuals in the network (network size), the number of connections each individual has to other individuals in the network (network connectedness), and whether all individuals in a network have the same (homogenous) or different (heterogenous) number of connections to other individuals in the network. We also varied whether the individuals asked their partners for help at random or in order of their livestock wealth. In virtually all of the simulations, herd survival was improved by selective asking compared to random asking.

The finding that selective asking leads to improvements - sometimes quite dramatic ones in herd survival compared to random asking may at first seem obvious given that real people do not typically interact with one another at random. However, what this finding demonstrates is that the success of a risk pooling system may be highly dependent upon the accuracy of the participants' knowledge regarding one another's wealth. Pastoralists may have an advantage in this regard because their wealth holdings are essentially public knowledge: it is difficult to hide a grazing cow. In societies where wealth comes in forms that are easier to keep private, 
it may be more difficult for need-based transfer systems to work, and risk management may need to take other forms. In future fieldwork we plan to compare the viability of need-based transfer systems among pastoral societies and societies in which wealth is more private.

In our model, we found that, given a homogenous network, increases in herd survival diminished when the size of the network got above ten. Also in a homogenous network, increases in herd survival diminished when each individual's number of connections got above 32. In a heterogenous network, however, the increase of an individual's herd survival diminished above the much lower number of 12 connections for selective asking ( 4 for random asking). The finding that, in homogenous networks, network sizes of more than ten did not lead to significant increases in herd survival shows that risk-pooling networks do not need to be large to be helpful. Small networks are significantly better than none at all, and large ones may not be necessary. This matches a finding regarding the usefulness of sharing among small groups of foragers arrived at analytically rather than through a simulation (Winterhalder 1990). The findings regarding network connectedness are particularly interesting because they yield predictions that could be easily tested with some additional fieldwork. Given that real world networks are likely to be heterogenous, the finding from the heterogenous network simulation that herd survival diminished when the number of connections reached about 12 is probably a better guide to reality than the finding from the homogenous network that this point was reached at about 32 .

Our simulations also suggest that a side effect of risk-pooling arrangements may be to help equalize the distribution of wealth among pastoralists, something that has been suspected for many years (e.g., Lewis, 1976:176). The most common way to compare degrees of inequality in the distribution of wealth is the Gini coefficient (Gini, 1912), which varies from zero, when all wealth holdings are equal, to one, when one person controls all the wealth. When agents in our simulations asked their partners for help in order of their wealth holdings, the result was a more equal distribution of wealth than when they asked for help at random, with Gini coefficients of 0.1659 and 0.2168 , respectively (see also Figure 9). Factors that work in the opposite direction include inheritance and a tendency for people in such societies to assort themselves by wealth not only residentially but also in terms of social and economic relationships, including riskpooling relationships (Borgerhoff Mulder et al., 2010). For example, when risk pooling partners 
are chosen among the Jie of Uganda and the Turkana of Kenya, "considerations of wealth enter into it, for a man does not wish to ally himself to someone considerably poorer than he is, and from whom, therefore, he may find difficulty in obtaining his due return and a fruitful relationship in general. A wealthy man never finds it difficult to make new bond-friends, but a poor man is usually acceptable only to similarly poor men" (Gulliver, 1955:210). The end result may be greater variation between than within pastoralist wealth strata, as is seen in at least some parts of East Africa (e.g., Cronk, 1990).

In future work we plan to systematically vary the nature of the randomness of disasters and the extent to which disasters are independent or correlated among individuals in a network. In our current model, disasters occur with exponentially distributed frequencies in time and with equal probability for each individual. As a result, there are correlations neither in time nor among neighbors in the networks for the disasters that they experience. This is clearly not the case for many disaster events in reality. Droughts are regional and hence affect neighbors simultaneously. They also tend to occur in multi-year periods. The same is often true for warfare and other types of adverse events that pastoralists experience. In future work we will study the influence of spatiotemporal correlations on the survival rates of risk-pooling networks. A clear and testable empirical implication of this is that herders might do well to seek out risk-pooling partners whose risk profiles differ from their own. It is possible that the topography of East Africa, which includes a great deal of local variability in altitude and hence in climate and rainfall, may make this relatively easy to accomplish. Existing ethnographic accounts of some East African pastoralists provide support for this idea. For example, Pokot men rarely have more than one stock friend in the same place, instead maintaining them in at least six to ten different places (Bollig 2006:287). Similarly, a Turkana herder will try to maintain bond-friendships in a variety of places to ensure that he has a potential supporter in most or all of the areas to which, in any year, he might wish to shift if his usual grazing areas happen to be in poor condition (Gulliver 1955: 212). Pastoralists living in more homogenous environments may have a more difficult time finding partners whose risk profiles differ from their own.

Risk pooling systems like the one explored in this article are often compared to insurance policies (e.g., Gurven et al. 2000). The similarity lies in the fact that both informal risk- 
pooling networks and insurance companies work by transferring risk from party to party. They also differ in many ways, most notably in terms of how the information and control over the system are distributed. The modern insurance industry is made possible by the ability to accumulate large amounts of data about rare events, which can then be used actuarially to calculate risks and set premiums (Bernstein, 1996; Levy, 2012). Informal risk-pooling systems, in contrast, rely upon knowledge about risk that is distributed among the participants and never formalized or centralized in any way. The contrast is similar to the one between centrally planned and unplanned economies (Hayek, 1945). In the former, the information needed to run the economy is accumulated and centralized, while in the latter it remains dispersed among the individual participants in the economy themselves. The use of distributed information combined with a decentralized system of asking for and transferring cattle may be a relatively efficient and effective informal risk management systems when formal insurance may not be viable for actuarial or institutional reasons.

Although this simulation was based primarily on a single ethnographic case, it may have implications for risk-pooling more generally, and particularly wherever environments are volatile enough to create uncertainty about the future availability of resources. Human subjects readily adopt a need-based transfer approach in the laboratory (Cronk \& Wasielewski 2008, Gazzillo et al. 2013), suggesting that this form of cooperation may be part of the human behavioral repertoire. However, it is clear that need-based transfers often manifest as a part of cultural systems that create shared norms of resource transfer such as the Maasai osotua system. The study of need-based transfer systems such as these may therefore lead to important insights about the interactions between the evolutionary forces favoring cooperation and cultural systems that enable coordination through shared expectations (Chwe 2001, Cronk \& Leech 2013).

Throughout evolutionary history, humans have lived in environments characterized by ecological uncertainty and resource scarcity. The pooling of risk through need-based transfer systems is one way of solving the adaptive problems associated with living in such environments and it is one that is necessarily dependent on the willingness of individuals to enter into trusting and cooperative risk pooling relationships. The results of the present model suggest that risk-pooling networks are particularly effective if individuals have the ability to selectively ask the most wealthy individuals in their networks and that risk pooling networks 
need neither to be large nor highly interconnected in order to be effective. Together these finding suggest that large and complex social insurance institutions are not necessary in order for humans to pool risk effectively. Simple risk pooling rules such as the osotua systems of the Maasai greatly enhance the ability of humans to survive and thrive in challenging ecological conditions. Need based transfers may therefore be a form of cooperation that is critical for understanding both the evolutionary and cultural adaptations that enabled human expansion into marginal environments.

\section{Acknowledgements}

This research was made possible by the support of the John Templeton Foundation grant "Generous by nature: Need-based transfers and the origins of human cooperation" to LC and CAA, National Science Foundation under grants no. BECS-1023101 and DMS-1313312 to DA and by a grant from the Volkswagen Foundation under the program on Complex Networks to DA and YH. This work was also supported by NSF under Grant No. SES-0345945 to the Decision Center for a Desert City (DCDC) at Arizona State University, and by National Institute of Healths Grant No. F32 CA144331 to CAA. The opinions expressed in this publication are those of the author(s) and do not necessarily reflect the views of the John Templeton Foundation, National Science Foundation or National Institutes of Health. We thank the Center for Advanced Study in the Behavioral Sciences at Stanford University, the Institute for Advanced Study, the Center for Theological Inquiry and the Center for Social Dynamics and Complexity at ASU for providing exciting intellectual environments that contributed to the genesis and synthesis of these ideas. We would also like to thank participants in the National Evolutionary Synthesis Center catalysis meeting for Synthesizing the Evolutionary and Social Science Approaches to Human Cooperation and the Cronk lab group for many insightful discussions and suggestions. Finally, we thank Daniel Hruschka, Marc Htt and Rolando de Aguiar for fruitful discussions and two anonymous reviewers for their comments that greatly improved the clarity of the manuscipt. Any opinions, findings and conclusions or recommendation expressed in this material are those of the author(s) and do not necessarily reflect the views of the National Science Foundation (NSF) or National Institute of Health (NIH).

\section{References}

Aktipis, C.A., Cronk, L., De Aguiar, R. (2011). Risk-pooling and herd survival: An agent-based model of a Maasai gift-giving system. Human Ecology, 39, 131-140.

Aktipis, C.A., Cronk, L., De Aguiar, R., In prep. Cooperation in an uncertain world: Need-based transfers outperform account keeping in volatile environments..

Almagor, U. (1978). Pastoral partners: Affinity and bond partnership among the Dassanetch of south-west Ethiopia. Manchester University Press. 
Barr, A., Genicot, G. (2008). Risk sharing, commitment, and information: An experimental analysis. Journal of the European Economic Association, 6(6), 1151-1185.

Bernstein, P. L. (1996). Against the Gods: The Remarkable Story of Risk. New York: John Wiley and Sons.

Blurton Jones, N. G. (1984). A selfish origin for human food sharing: tolerated theft. Ethology and sociobiology 5(1), 1-3.

Blurton Jones, N. G. (1987). Tolerated theft: Suggestions about the ecology and evolution of sharing, hoarding, and scrounging Social Science Information 26, 31-54. Reprinted (1991) In Primate politics, eds. G. Schubert \& R. Masters, Southern Illinois University Press, 170-187.

Bollig, M. (1998). Moral economy and self-interest. Kinship, friendship, and exchange among the Pokot (N.W. Kenya). In: Schweizer T., White, D. (Eds.), Kinship, Networks, and Exchange. Cambridge University Press, Cambridge, pp. 137-157.

Bollig, M. (2006). Risk Management in a Hazardous Environment: A Comparative Study of Two Pastoral Societies. Springer, New York.

Borgerhoff Mulder, M., Fazzio, I., Irons, W., McElreath, R.L., Bowles, S., Bell, A., Hertz, T., Hazzah, L. (2010). Pastoralism and wealth inequality: Revisiting an old question. Current Anthropology, 51(1), 35-48.

Borgerhoff Mulder, Monique, and Bret A. Beheim. (2011). Understanding the Nature of Wealth and Its Effects on Human Fitness. Philosophical Transactions of the Royal Society B: Biological Sciences 366, 344-356. doi:10.1098/rstb.2010.0231.

Cashdan, E.A. (1985). Coping with risk: Reciprocity among the Basarwa of northern Botswana. Man (N. S.), 20, 454-474.

Chwe, M. S. Y. (2001). Rational ritual: Culture, coordination, and common knowledge. Princeton University Press, Princeton, NJ.

Cosmides, L., Tooby, J. (2005). Neurocognitive adaptations designed for social exchange. In: Buss, D.M. (Eds.), The Handbook of Evolutionary Psychology. Wiley, New York, 584-627.

Cronk, L. (1990). Stratification, bridewealth, and marriage patterns among the Mukogodo and their neighbors, Laikipia District, Kenya. Research in Economic Anthropology, 12, 89-109.

Cronk, L. (2004). From Mukogodo to Maasai: Ethnicity and Cultural Change in Kenya. Boulder, CO: Westview Press.

Cronk, L. (2007). The influence of cultural framing on play in the Trust Game: A Maasai example. Evolution and Human Behavior, 28, 352-358.

Cronk, L., Wasielewski, H. (2008). An unfamiliar social norm rapidly produces framing effects in an economic game. Journal of Evolutionary Psychology, 6(4), 283-308.

Cronk, L., Leech, B. (2013). Meeting at Grand Central: Understanding the Social and Evolutionary Roots of Cooperation. Princeton University Press, Princeton, NJ.

Dahl, G., Hjort, A. (1976). Having Herds: Pastoral Herd Growth and Household Economy. University of Stockholm, Stockholm.

De Vries, D., Leslie, P.W., McCabe, J.T. (2006). Livestock acquisitions dynamics in nomadic pastoralist herd demography: A case study among Ngisonyoka herders of South Turkana, Kenya. Human Ecology, 34(1), 1-25. 
Dorfman, M.A. (2007). Introduction to Risk Management and Insurance, ninth edition. PrenticeHall, Saddle River, NJ.

Dyson-Hudson, N. (1966). Karimojong politics. Clarendon.

Fafchamps, M., Lund, S. (2003). Risk-sharing networks in rural Philippines. Journal of Development Economics, 71, 261-287.

Flannery, K.V., Marcus, J., Reynolds, R.G. (1989). Flocks of the Wamani: A Study of Llama Herders on the Punas of Ayacucho, Peru. Academic Press, Inc, New York.

Galaty, J. G. (1982). Being "Maasai"; being "people-of-cattle": Ethnic shifters in East Africa. American Ethnologist, 9(1), 1-20.

Gazzillo, S., Sopher, B., Aktipis, C. A. \& Cronk, L. (2013). The origins of risk sharing: an experimental approach. Working paper, Department of Economics, Rutgers University.

Gerkey, A. P. (2010). From State collectives to local commons: cooperation and collective action among salmon fishers and reindeer herders in Kamchatka, Russia. Doctoral dissertation, Rutgers University.

Gini, C. (1912). "Italian: Variabilit e mutabilit" (Variability and Mutability', C. Cuppini, Bologna, 156 pages. Reprinted in Memorie di metodologica statistica (Ed. Pizetti E, Salvemini, T). Rome: Libreria Eredi Virgilio Veschi (1955).

Gulliver, P.H. (1955). The Family Herds: A Study of Two Pastoral Tribes in East Africa The Jie and Turkana. Routledge \& Kegan Paul, London.

Gurven, M., Allen-Arave, W., Hill, K., Hurtado, M. (2000). It's a Wonderful Life: signaling generosity among the Ache of Paraguay. Evolution and Human Behavior, 21, 263-282.

Gurven, M., Hill, K., \& Jakugi, F. (2004). Why do foragers share and sharers forage? Explorations of social dimensions of foraging. Research in Economic Anthropology, 23, 19-43.

Gurven, M., Hill, K. (2009). Why do men hunt? A reevaluation of "Man the Hunter" and the Sexual Division of Labor. Current Anthropology, 50(1), 51-74.

Hayek, F.A. (1945). The Use of Knowledge in Society. The American Economic Review, 35(4), 519-530.

Hegmon, M. (1989). Risk reduction and variation in agricultural economies: A computer simulation of Hopi agriculture. Research in Economic Anthropology, 11, 89-121.

Isaac, G. L. (1978). Food sharing and human evolution: archaeological evidence from the PlioPleistocene of east Africa. Journal of anthropological research 34, 311-325.

Jaeggi, A. V., \& van Schaik, C. P. (2011). The evolution of food sharing in primates. Behavioral Ecology and Sociobiology, 65(1), 2125-2140.

Levy, J. (2012). Freaks of Fortune: The Emerging World of Capitalism and Risk in America. Harvard University Press, Cambridge, MA.

Lewis, I.M. (1976). Social Anthropology in Perspective. Cambridge University Press, Cambridge.

Mace, R. (1993). Nomadic Pastoralists Adopt Subsistence Strategies That Maximise Long-Term Household Survival. Behavioral Ecology and Sociobiology 33: 329-334. 
Mace, R. (1996). When to Have Another Baby: A Dynamic Model of Reproductive Decision-Making and Evidence from Gabbra Pastoralists. Ethology and Sociobiology 17: 263-273.

Mace, R., and Houston, A. (1989). Pastoralist strategies for survival in unpredictable environments: a model of herd composition that maximizes household viability. Agricultural Systems 31:185204.

McCabe, J.T. (1990). Success and failure: The breakdown of traditional drought coping institutions among the Turkana of Kenya. Journal of Asian and African Studies, 25, 146-160.

Peterson, N. (1993). Demand sharing: Reciprocity and the pressure for generosity among foragers. American Anthropologist 95(4):860-874.

Potkanski, T. (1999). Mutual assistance among the Ngorongoro Maasai. In: Anderson D. M., Broch-Due, V. (Eds.), The Poor Are Not Us: Poverty and Pastoralism in Eastern Africa. John Currey, Oxford, 199-217.

Price, J.A. (1962). Washo Economy. Nevada State Museum Anthropological Papers, 6.

Price, J.A. (1975). Sharing: The integration of intimate economies. Anthropologica, n.s., 17, 3-27.

Watts, D.J., Strogatz, S.H. (1998). Collective dynamics of 'small-world' networks. Nature, 393(6684), 409-10.

Wiessner, P. (1982). Beyond willow smoke and dogs' tails: A comment on Binford's analysis of hunter-gatherer settlement systems. American Antiquity, 47(1), 171-178.

Wiessner, P. (2002). Hunting, healing, and hxaro exchange: A long-term perspective on !Kung (Ju/'hoansi) large-game hunting. Evolution and Human Behavior, 23, 407-436.

Winterhalder, B. (1986). Diet choice, risk, and food sharing in a stochastic environment. Journal of Anthropological Archaeology 5, 369-392.

Winterhalder, B. (1990). Open field, common pot: Harvest variability and risk avoidance in agricultural and foraging societies. In E. A. Cashdan (Ed.), Risk and Uncertainty in Tribal and Peasant Economies. Westview Press, Boulder, CO, 67-87.

Winterhalder, B. (2007). Risk and decision-making. In R. I. M. Dunbar \& L. Barrett (Eds.), Oxford Handbook of Evolutionary Psychology. Oxford University Press, Oxford, 433-445.

Woodburn, J. (1998). Sharing is not a form of exchange: An analysis of property sharing in immediate-return hunter-gatherer societies. In: Hann, C. (Eds.), Property Relations: Renewing the Anthropological Tradition. Cambridge University Press, Cambridge, 48-63. 


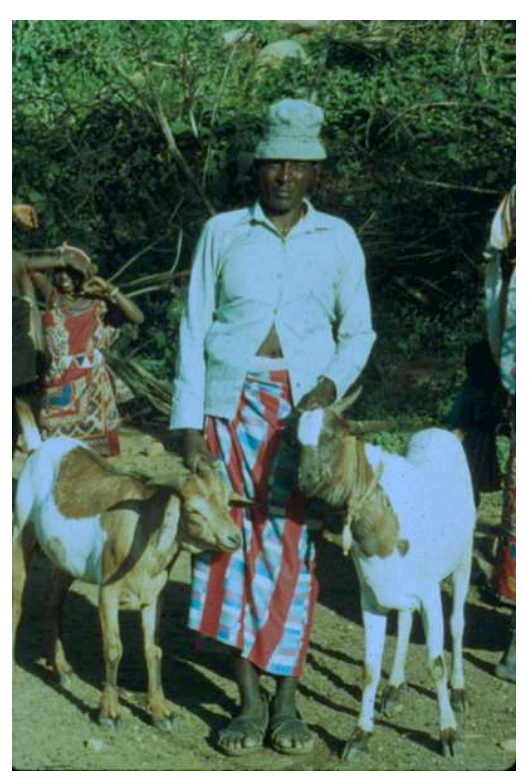

Figure 1: A Maasai man posing with his favorite goats.
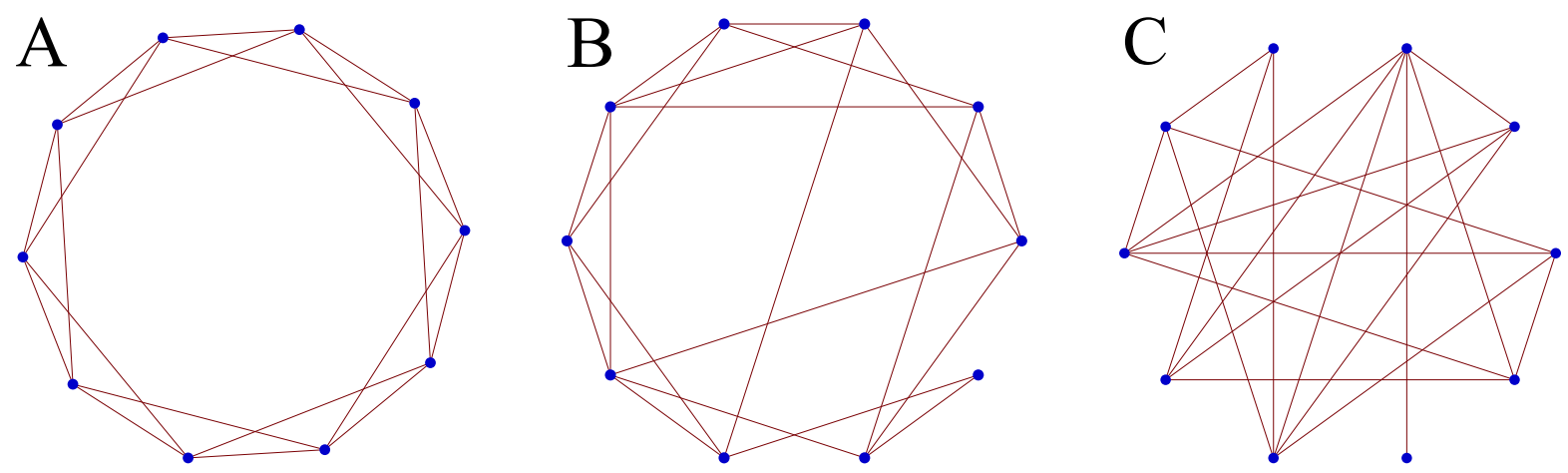

Figure 2: (A) A homogeneous (non-random) network with parameters: $N=10, k=4, \beta=0,(\mathrm{~B})$ a Watts-Strogatz (heterogeneous) network with $\beta=0.2$, and (C) a Watts-Strogatz network with $\beta=1$. 


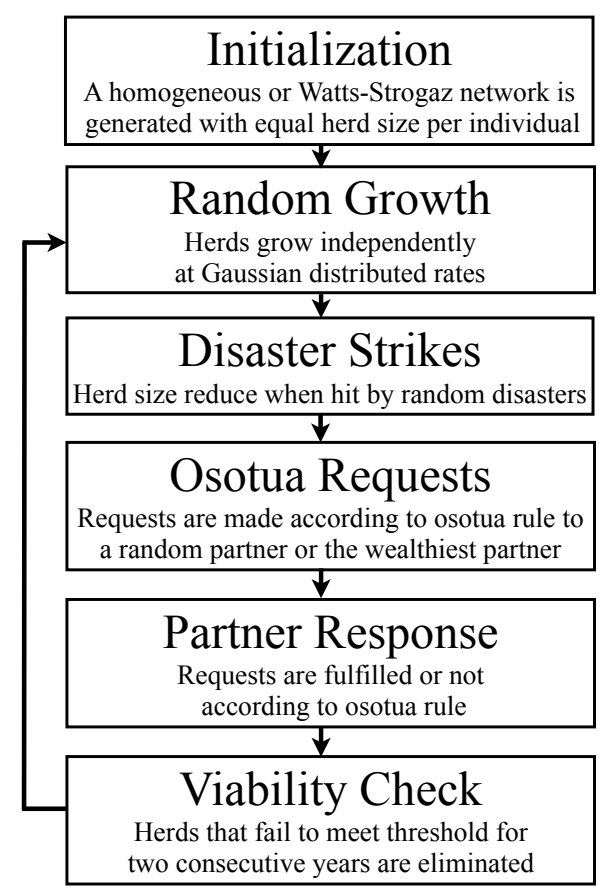

Figure 3: Flow chart of steps in simulation of osotua gift giving on a homogeneous or Watts-Strogatz network.
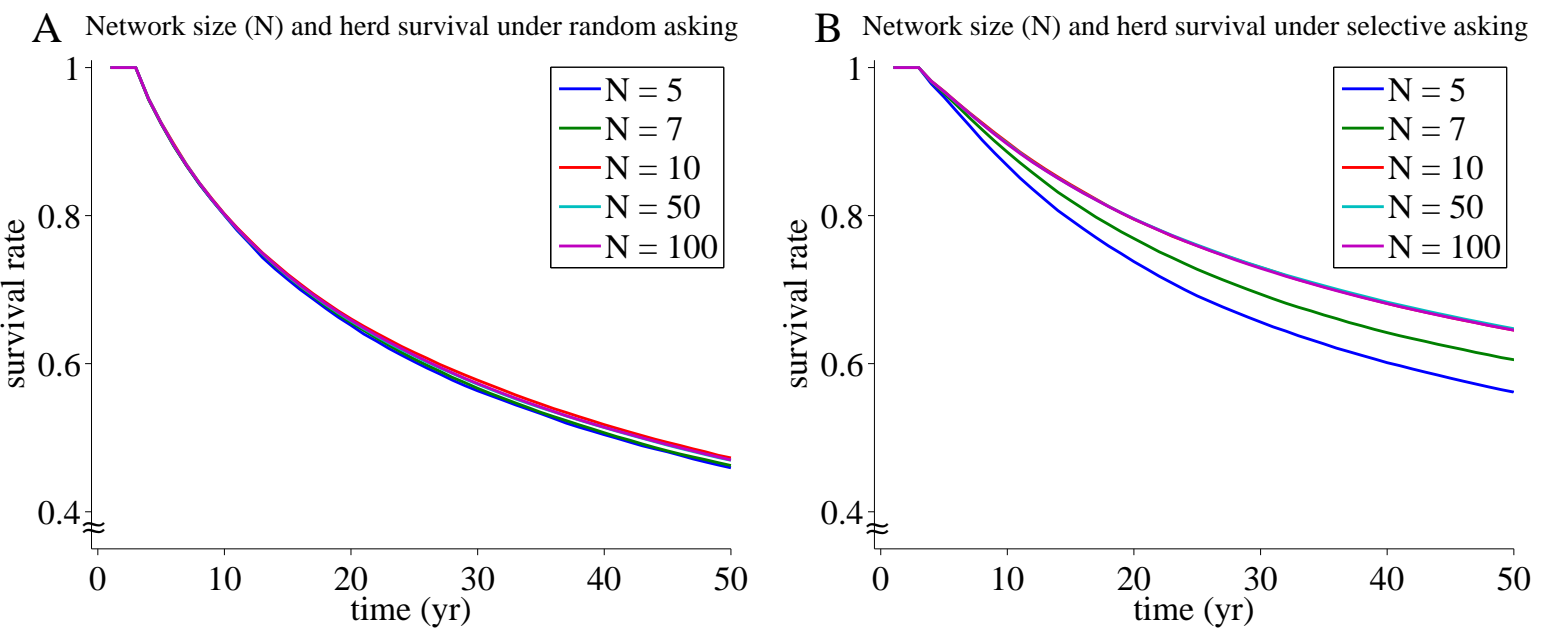

Figure 4: Varying network size $N$ for a constant number of connections $(k=4)$ and a homogeneous network. (A) When individuals in need randomly ask one of their partners for help, $N$ shows little effect on herd survival. (B) When individuals in need ask their partner with the largest herd, increasing the network size $N$ improves herd survivorship for $N \leq 10$. 

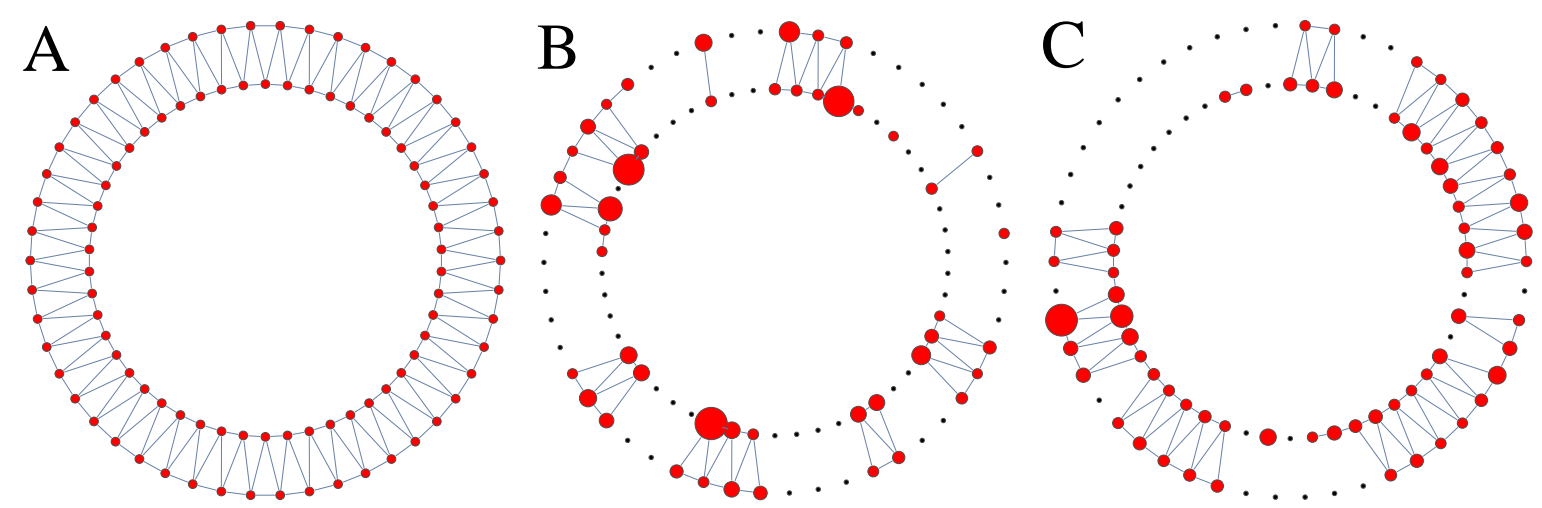

Figure 5: Topology of the osotua networks. Red circles indicate individuals with surviving herds and the size of the circle indicates their corresponding herd size in year 50. Black dots indicate individuals whose herds have been eliminated from the simulation because they dropped below the viability threshold in two consecutive years. (A) The initial homogeneous osotua network where every individual is the partner of his closest 4 neighbors. (B) A typical topology of the same network in year 50 using a random asking strategy. (C) A typical topology of the same network in year 50 using a selective asking strategy.

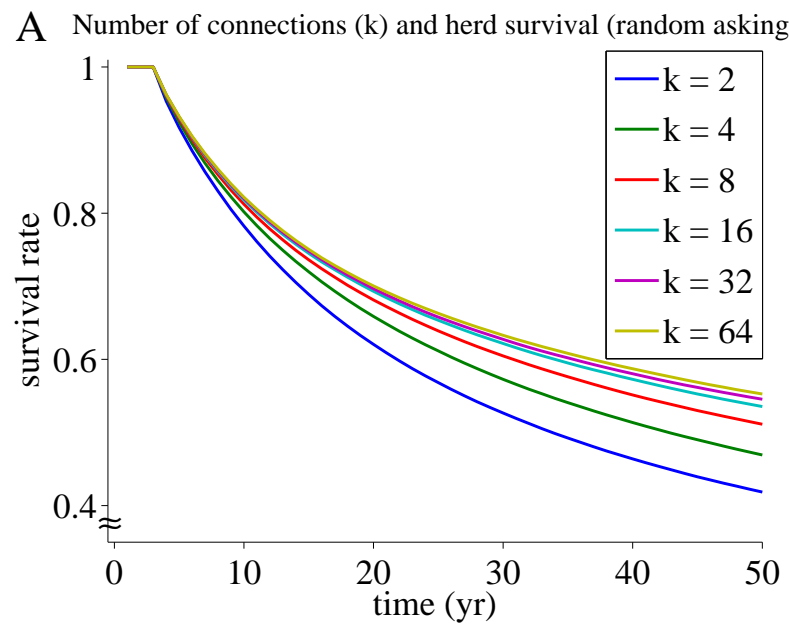

B Number of connections (k) and herd survival (selective asking)

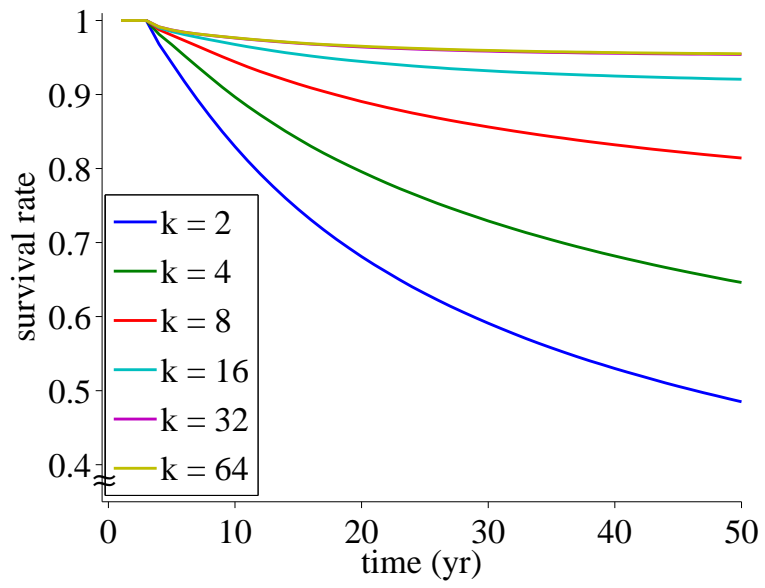

Figure 6: Herds have a better chance to survive when individuals have more connections $k$ for homogenous $(\beta=0)$ networks of size $N=100$. The marginal benefit to additional connections $k$ drops off quickly as $\mathrm{k}$ increases. (A) random asking (B) asking the partner with the largest herd. 
Randomness $(\beta)$ and herd survival in year 50

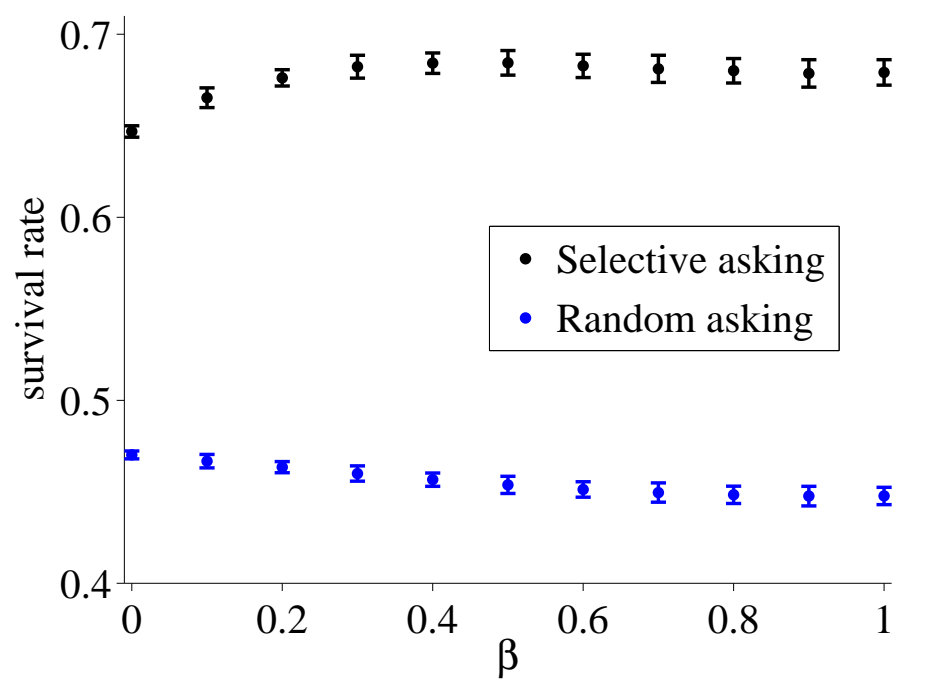

Figure 7: Average survival rates after 50 years (dots) and their standard deviations (error bars) as a function of network randomness $(\beta)$. Random asking (blue dots) leads to a decrease in the survival rate as $\beta$ increases to about 0.5 . Selective asking(black dots) leads to an increase in the survival rate as $\beta$ increases up to about 0.3 . The network size remains constant at $N=100$ and the number of connections is constant at $k=4$.

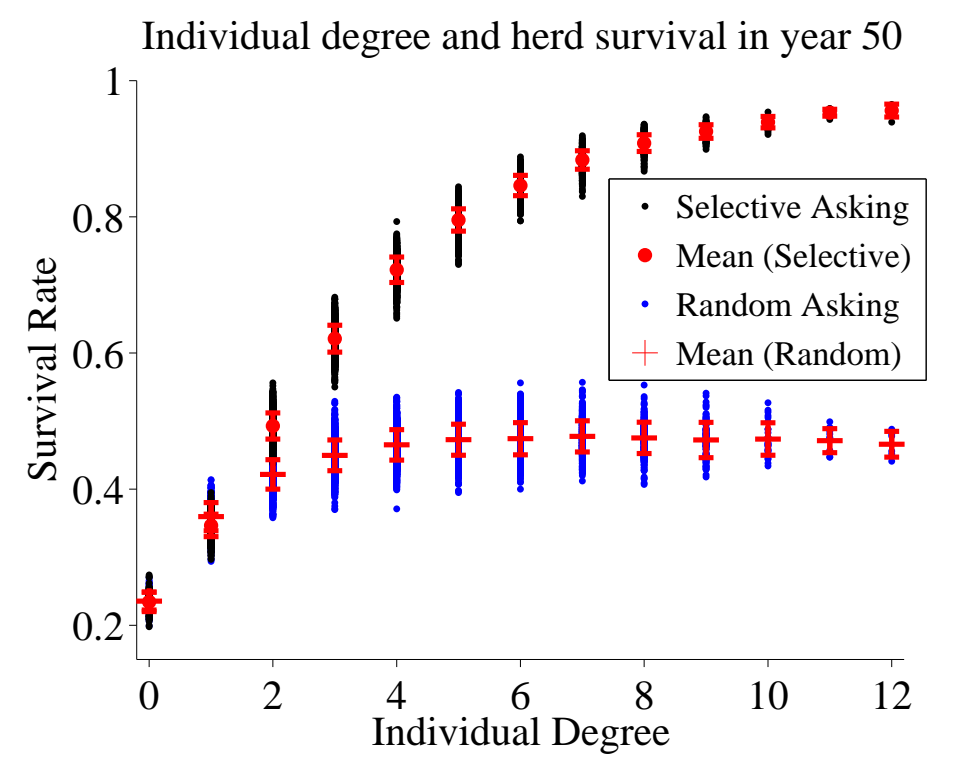

Figure 8: Individual survival rate as a function of the number of initial osotua partners (degree) for random (blue dots) and selective (black dots) asking. The network size and the average number of connections are constant $(N=100, k=4)$ and $\beta=0.8$. The data represent averages and standard deviations (red symbols) for 1000 simulation runs for each of a 1000 randomly generated topologies. 


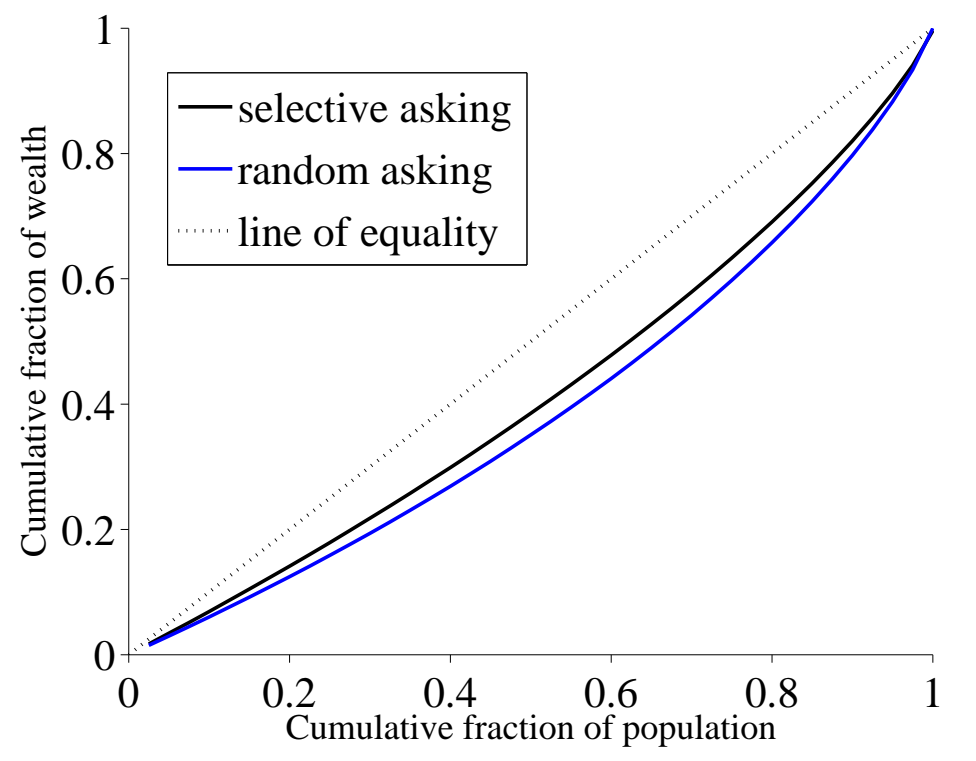

Figure 9: Lorenz curves showing the cumulative distribution of wealth (measured by the number of cattle owned) as a function of the fraction of the population. The diagonal curve (dotted line) represents a completely uniform wealth distribution. Random asking (blue curve) leads to a more uneven wealth distribution than selective asking. 